\title{
A Case Report of a Solitary Pelvic Mass Proven to Be a Lymph Nodal Metastasis from Anal Cancer
}

\author{
Katsuyuki Sakanaka Takashi Mizowaki \\ Department of Radiation Oncology and Image-Applied Therapy, Graduate School of \\ Medicine, Kyoto University, Kyoto, Japan
}

\section{Keywords}

Solitary pelvic-wall mass · Anal cancer $\cdot{ }^{18} \mathrm{~F}$-fluorodeoxyglucose positron emission tomography · Surgical tract recurrence $\cdot$ Chemoradiotherapy

\begin{abstract}
A solitary pelvic-wall lymph nodal metastasis can be mistaken as a primary malignancy when a primary tumor has not been diagnosed. We report the case of a 72-year-old woman with a solitary left pelvic-wall mass that was finally proven to be a left internal iliac lymph nodal metastasis from anal cancer. No signs of the primary tumor had been initially found by general screening using computed tomography, colonoscopy, pelvic magnetic resonance imaging, and gynecological/urological examination; however, squamous cell carcinoma was detected by surgical biopsy of the left pelvic-wall mass. Additional ${ }^{18} \mathrm{~F}$-fluorodeoxyglucose positron emission tomography $\left({ }^{18} \mathrm{~F}-\mathrm{FDG}-\mathrm{PET}\right)$ showed focal accumulations in the left pelvic mass and the anal canal. A biopsy of the induration in the anal canal led to the diagnosis of anal cancer, clinical T2N2M0, and stage IIIB (UICC-TNM 7th ed.), which was indicated for definitive chemoradiotherapy. Two months after completing a definitive chemoradiotherapy for anal cancer, a fixed induration developed under the surgical wound along with the surgical tract of the biopsy site. Physical examination and ${ }^{18} \mathrm{~F}$-FDG-PET/computed tomography led to the clinical diagnosis of unresectable surgical tract recurrence of anal cancer. The patient underwent palliative treatment and died 14 months after the diagnosis of the surgical tract recurrence. In conclusion, anal cancer may present as a solitary pelvic mass without any anal symptoms. To evaluate the solitary pelvic mass, ${ }^{18} \mathrm{~F}-\mathrm{FDG}-\mathrm{PET} / \mathrm{computed}$ tomography, along with digital ex-
\end{abstract}


Sakanaka and Mizowaki: A Solitary Pelvic Mass Proven to Be a Lymph Nodal Metastasis from Anal Cancer

amination, will probably help in establishing an accurate diagnosis. Anal cancer must be considered during the differential diagnosis of a solitary pelvic-wall mass for a correct diagnosis and to avoid unnecessary procedures.

\section{Introduction}

A solitary pelvic-wall lymph nodal metastasis may be unintentionally misdiagnosed as a primary malignancy when screening fails to detect a true primary tumor. A biopsy of a solitary pelvic mass should be performed to confirm its histology. The risk of a surgical biopsy is limited; however, surgical biopsy of a pelvic mass may cause tumor implantations along the surgical wound and tract. Here we report the case of a surgical tract recurrence after surgical biopsy of a solitary pelvic mass, which was finally proven to be a solitary left internal iliac nodal metastasis of anal squamous cell carcinoma.

\section{Case Presentation}

A 72-year-old woman was hospitalized because of acute cerebral ischemia. A routine examination incidentally showed mild anemia with a positive fecal blood test. She underwent esophagogastroduodenoscopy, colonoscopy, and computed tomography to rule out a gastrointestinal malignancy. The esophagogastroduodenoscopy and colonoscopy did not detect any abnormal findings at that time. Computed tomography identified a solitary mass of $4 \mathrm{~cm}$ located on the left pelvic wall. Gynecological and urological examinations did not find any findings suspicious for a malignancy. The patient was referred to our hospital to undergo further evaluation 4 months after the initial detection of the positive fecal blood test. Computed tomography and magnetic resonance imaging were reperformed at our hospital, the findings of which were compatible with the previous ones (Fig. 1).

A soft-tissue tumor or lymphoma was clinically suspected. To select a definitive treatment and obtain histological confirmation, en bloc resection after surgical biopsy was planned. Intraoperative consultation of the surgical biopsy specimen led to the diagnosis of squamous cell carcinoma, and the planned en bloc tumor resection was canceled. The patient underwent ${ }^{18} \mathrm{~F}$-fluorodeoxyglucose positron emission tomography $\left({ }^{18} \mathrm{~F}-\mathrm{FDG}-\mathrm{PET}\right)$, which showed abnormal accumulations in the left pelvic tumor and the anal canal (Fig. 2). Digital examination detected an induration along the anal canal. Colonoscopy showed an elevated lesion located between the anal canal and the rectum (Fig. 2). The histology of the elevated lesion was squamous cell carcinoma. A diagnosis of anal squamous cell carcinoma, clinical stage IIIB (T2N2M0) based on the Union for International Cancer Control TNM staging, 7th edition, was established 7 months after the blood-positive fecal test.

Two months after the completion of standard chemoradiotherapy for anal cancer, the patient noticed a fixed induration beneath the scar of the surgical biopsy of the left pelvic mass. ${ }^{18}$ F-FDG-PET and magnetic resonance imaging showed the development of a solitary mass along the surgical tract of the biopsy, while the abnormal accumulation in the primary anal tumor and left iliac nodal metastasis had disappeared (Fig. 3). A multidisciplinary team established a clinical diagnosis of inoperable surgical tract recurrence and initiated palliative chemotherapy and radiotherapy for the implanted tumor. The patient died from an uncontrollable infection of the fistula among the surgical tract recurrence, descending colon, and abdominal wall 14 months after the diagnosis of surgical tract recurrence. 
Sakanaka and Mizowaki: A Solitary Pelvic Mass Proven to Be a Lymph Nodal Metastasis from Anal Cancer

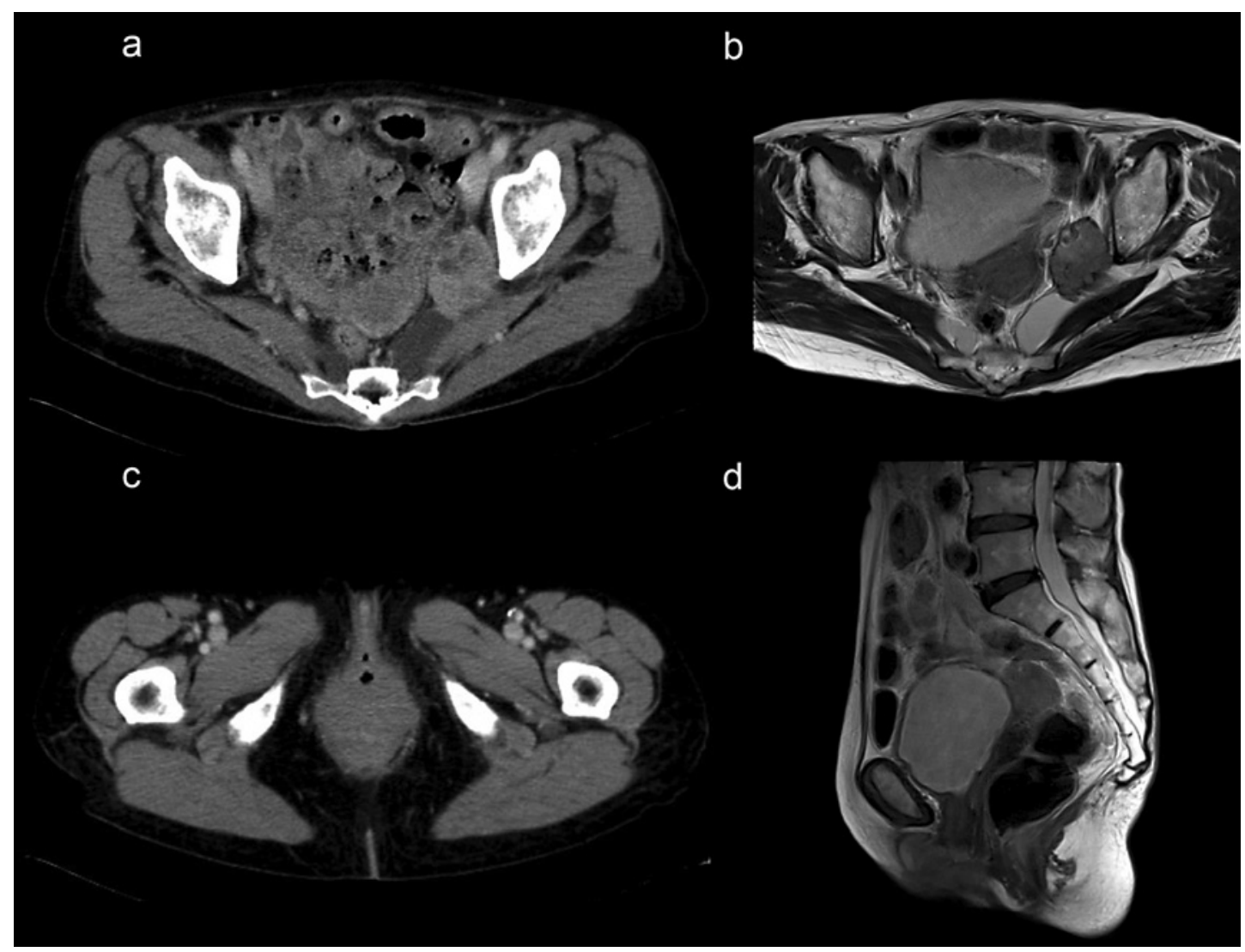

Fig. 1. Computed tomography with contrast enhancement (a) and T2-weighted axial magnetic resonance imaging (b) led to the detection of a 4-cm mass on the pelvic wall. Computed tomography with contrast enhancement (c) and T2-weighted sagittal magnetic resonance imaging (d) did not detect apparent anal lesions.

\section{Discussion}

Two important clinical observations were made in the present case. First, a lymph nodal metastasis from anal cancer may present as a solitary pelvic mass. Second, surgical tract recurrence may occur after a surgical biopsy for pelvic lymph nodal metastasis of an anal cancer.

Most patients with anal cancer have some complaints at diagnosis; however, approximately $20-33 \%$ of patients with anal cancer do not show any symptoms at diagnosis [1]. If an anal primary cancer is not detected, a solitary pelvic lymph nodal metastasis may present as a primary tumor in patients with anal cancer. Metastasis from anal cancer occurs most commonly in the mesorectal lymph nodes, with inguinal nodal spread being the second most common location of nodal metastasis. The incidence of a solitary internal iliac nodal metastasis, such as the one presented by the current case, is $1 \%$ [2]. The unusual solitary lymph nodal metastasis prevented the establishment of a correct diagnosis.

Peritoneal seeding is extremely rare after definitive chemoradiotherapy for anal cancer. To our knowledge, no report has mentioned peritoneal seeding after chemoradiotherapy for anal cancer. In the current case, the time course from surgical biopsy to surgical tract recurrence, and the presence of a cord-like structure from the recurrence site to the surgical wound, indicated that the recurrence was probably associated with the surgical manipu- 
Sakanaka and Mizowaki: A Solitary Pelvic Mass Proven to Be a Lymph Nodal Metastasis from Anal Cancer

Fig. 2. a A tumor was detected in the anal canal by colonoscopy. b, c Abnormal accumulations in the left internal iliac lymph node and anal canal on ${ }^{18} \mathrm{~F}$-fluorodeoxyglucose positron emission tomography.

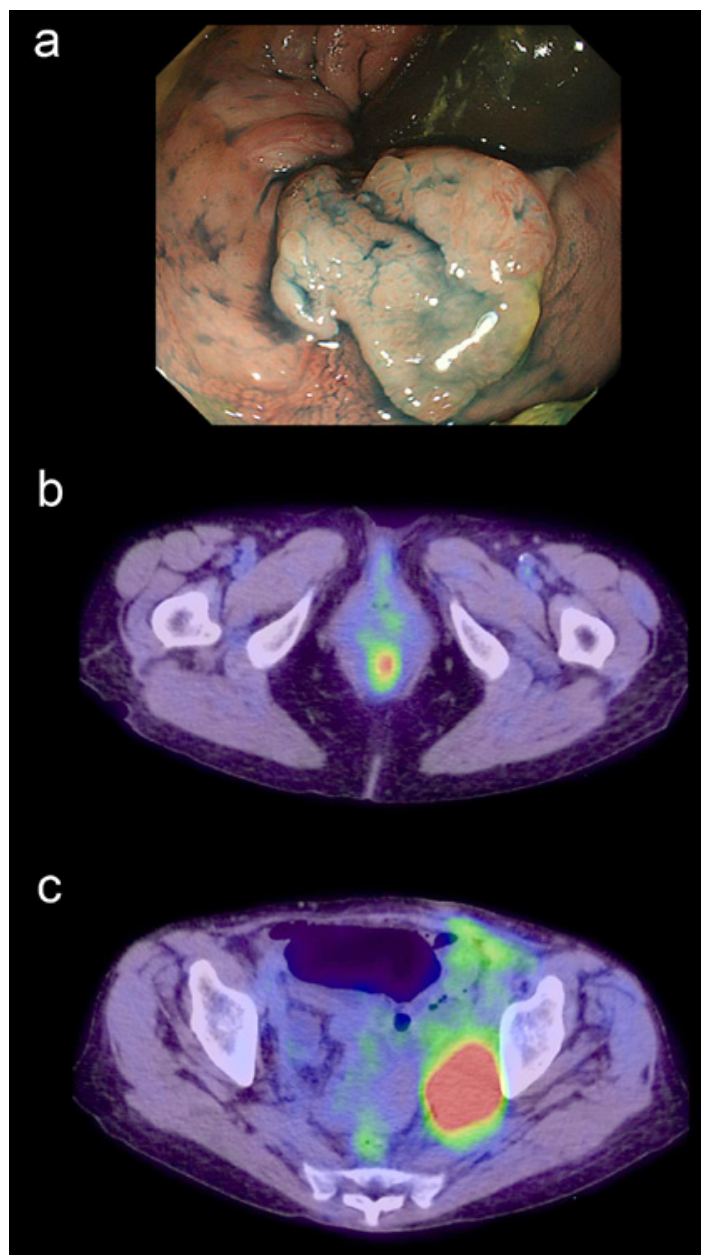

lation. Surgical wound recurrences rarely occur after surgery for gastrointestinal cancers. The estimated incidence of surgical wound recurrences after open surgery for gastrointestinal cancer is $0.6-2.2 \%$ [3-5]. Locally, anal squamous cell carcinoma is a chemosensitive and curable disease by definitive chemoradiotherapy $[6,7]$; however, surgical tract recurrence is difficult to control if it occurs. Unnecessary manipulations for a suspected tumor should be avoid.

${ }^{18} \mathrm{~F}$-FDG-PET is useful in detecting a primary tumor in anal cancer. A review article reported that ${ }^{18} \mathrm{~F}-\mathrm{FDG}-\mathrm{PET} / \mathrm{computed}$ tomography has a sensitivity of $89-100 \%$ for the detection of primary tumors of anal cancer, whereas computed tomography has a sensitivity of $58-75 \%$ [8]. Digital examination is also a specific tool to detect anal lesions. ${ }^{18}$ F-FDG-PET/computed tomography with digital examination before a surgical biopsy probably would have helped avoid the biopsy procedure in the current patient. ${ }^{18} \mathrm{~F}$-FDG$\mathrm{PET} /$ computed tomography and digital examination is recommended for the evaluation of a solitary pelvic tumor in order to reduce the risk of unnecessary biopsy of pelvic lymph nodes.

In conclusion, anal cancer may present as a solitary pelvic mass without any anal symptoms. To evaluate the solitary pelvic mass, ${ }^{18} \mathrm{~F}$-FDG-PET/computed tomography with digital examination will probably help in the establishment of a correct diagnosis. Anal cancer should be considered in the differential diagnosis of a solitary pelvic mass. 


\section{Case Reports in \\ Oncology}

\begin{tabular}{l|l}
\hline Case Rep Oncol 2020;13:164-169 \\
\hline DOI: 10.1159/000505969 & $\begin{array}{l}\odot \text { 2020 The Author(s). Published by S. Karger AG, Basel } \\
\text { www.karger.com/cro }\end{array}$ \\
\hline
\end{tabular}

Sakanaka and Mizowaki: A Solitary Pelvic Mass Proven to Be a Lymph Nodal Metastasis from Anal Cancer

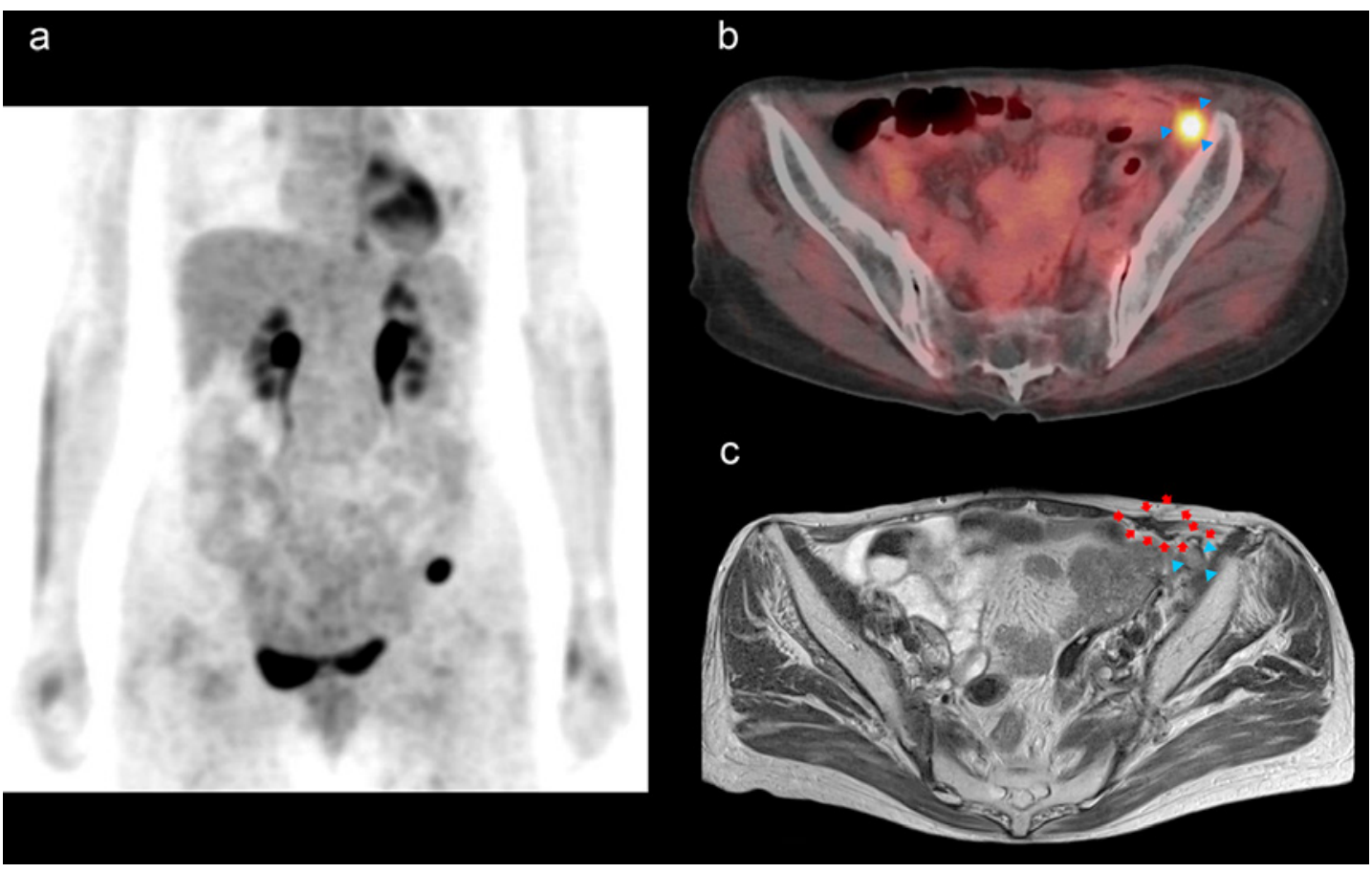

Fig. 3. Surgical tract recurrence after chemoradiotherapy. a An abnormal focal accumulation was present only in the left lower quadrant at maximal intensity projection on ${ }^{18} \mathrm{~F}$-fluorodeoxyglucose positron emission tomography. b Axial ${ }^{18} \mathrm{~F}$-fluorodeoxyglucose positron emission tomography image. c T2-weighted axial magnetic resonance imaging. The blue arrowheads indicate the gross recurrent tumor. The red arrows indicate a subcutaneous surgical wound scar and a cord-like structure in the abdominal cavity. The surgical wound scar and the cord-like structure were connected to the recurrent tumor.

\section{Acknowledgements}

The authors would like to thank Enago (http://www.enago.jp) for the English language review.

\section{Statement of Ethics}

This study was conducted in accordance with the Declaration of Helsinki and the Ethical Guideline for Medical and Health Research Involving Human Subjects in Japan. General written consent for the use of clinical data for research purposes was obtained from the patient before starting radiotherapy.

\section{Disclosure Statement}

The authors have no conflicts of interest. 


\section{Funding Sources}

This work was partially supported by Grants-in-Aid for Scientific Research from the Ministry of Education, Culture, Sports, Science, and Technology of Japan under Grant No. 17 K16434.

\section{Author Contributions}

Katsuyuki Sakanaka participated in the study design, acquired data, and drafted the manuscript. Takashi Mizowaki provided manuscript writing assistance. Both authors read and approved the final manuscript.

\section{References}

1 Osborne MC, Maykel J, Johnson EK, Steele SR. Anal squamous cell carcinoma: an evolution in disease and management. World J Gastroenterol. 2014;20(36):13052-9.

2 Kuehn PG, Eisenberg H, Reed JF. Epidermoid carcinoma of the perianal skin and anal canal. Cancer. 1968; 22(5):932-8.

3 Reilly WT, Nelson H, Schroeder G, Wieand HS, Bolton J, O'Connell MJ. Wound recurrence following conventional treatment of colorectal cancer. A rare but perhaps underestimated problem. Dis Colon Rectum. 1996; 39(2):200-7.

4 Duarte I, Llanos O. Patterns of metastases in intestinal and diffuse types of carcinoma of the stomach. Hum Pathol. 1981;12(3):237-42.

5 Fortner JG, Lawrence W Jr. Implantation of gastric cancer in abdominal wounds. Ann Surg. 1960;152:789-94.

6 Sakanaka K, Itasaka S, Ishida Y, Fujii K, Horimatsu T, Mizowaki T, et al. Dosimetric advantages and clinical outcomes of simultaneous integrated boost intensity-modulated radiotherapy for anal squamous cell carcinoma. Radiat Oncol J. 2017;35(4):368-79.

7 Sakanaka K, Ishida Y, Mizowaki T. A case report of locally advanced anal cancer with solitary cutaneous nodular metastasis in the ipsilateral labia majora treated with definitive chemoradiotherapy. Case Rep Oncol. 2019;12(3):721-7.

8 Agarwal A, Marcus C, Xiao J, Nene P, Kachnic LA, Subramaniam RM. FDG PET/CT in the management of colorectal and anal cancers. AJR Am J Roentgenol. 2014;203(5):1109-19. 